PRESENZA DI BACILLUS CEREUS, ESCHERICHIA COLI E ENTEROBACTERLACEAE IN RICOTTA FRESCA E SALATA: CONTROLLI UFFICIALI NEL PERIODO 2009 2012.

\title{
Presence of Bacillus cereus, Escherichia coli and Enterobacteriaceae in fresh and salted Ricotta cheese: official controls in Sardinia during the period 2009 $-2012$.
}

\author{
Fadda Antonio*, Delogu Alida, Mura Elia, Noli Alessia Caterina, Porqueddu Giuseppina, Rossi Maria \\ Lucia, Terrosu Giovanni \\ *Corresponding author. Tel: (+39) 792892352 ; Fax: (+39)2892324 E-mail: Antonio.fadda@izs- \\ sardegna.it \\ Dipartimento di Igiene degli Alimenti, Istituto Zooprofilattico Sperimentale della Sardegna, Sassari, \\ Italia
}

\begin{abstract}
Results of microbiological official analysis carried out during 2009-2012 for detection of Bacillus cereus, Escherichia coli and Enterobacteriaceae in fresh and salted Ricotta cheese marketed in Sardinia, are reported. The aim of this research was to evaluate the presence of contaminants indicators of process hygiene, and the presence of B. cereus, which while not covered in the EC Regulation 2073/05 for this product, it may represent a risk as a potential producer of toxins harmful to the consumer health. Were analyzed a total of 157 samples according to ISO reference. The results indicate a widespread presence of the three types of microorganisms sought: prevalence of B. cereus $53 \%, 23 \%$ Enterobacteriaceae, E. coli $12 \%$. In conclusion, it is desirable the introduction, in the production process, keeping the cold chain to prevent the risk from $B$. cereus, and improved hygiene of processing plant, equipment and personnel, to reduce recontamination of the product by the Enterobacteriaceae and E. coli.
\end{abstract}

Keywords: Ricotta cheese, Bacillus cereus, Escherichia coli, Enterobacteriaceae.

\section{INTRODUZIONE}

La Ricotta, fresca e salata, rappresenta, grazie alla sua maggiore diffusione rispetto al passato, una risorsa economica importante per l'industria lattiero-casearia. Dati dell'Istituto di Servizi per il Mercato Agricolo Alimentare (ISMEA), relativi al primo trimestre del 2012, ne confermano un trend crescente di produzione e consumo, evidenziando un aumento dei prezzi pari al $14 \%$ rispetto all'anno passato. Il processo produttivo prevede il riscaldamento ad elevate temperature (variabili fra 70 e $85^{\circ} \mathrm{C}$ ) del siero di latte residuato dal processo di caseificazione, che porta alla coagulazione delle sieroproteine. Dopo l'affioramento, il prodotto viene raccolto e fatto sgrondare in fiscelle monouso (ricotta fresca) oppure pressato in stampi, salato a secco e stagionato per un breve periodo, variabile dai 5 ai 15 giorni (ricotta salata). Durante la fase di produzione la temperatura raggiunta è tale da eliminare i microrganismi patogeni e contaminanti presenti nel siero, ma non le spore di alcuni tipi di microrganismi come Bacillus cereus, che in certe condizioni (raffreddamento troppo lento del prodotto) possono germinare, passare alla forma vegetativa e dare luogo alla produzione di tossine. Nella ricotta si riscontrano spesso contaminazioni da parte di questo microrganismo, che trova in questo prodotto un ottimo substrato di crescita, soprattutto in condizioni di conservazione di abuso termico. Uno studio condotto sulla contaminazione da Bacillus cereus dei prodotti lattiero-caseari della Sardegna ha evidenziato una prevalenza di contaminazione della ricotta del $78 \%$, con livelli di

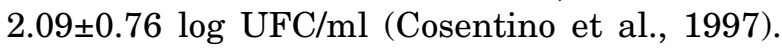
La presenza invece di altri contaminanti nel 
prodotto (Escherichia coli e Enterobacteriaceae), in particolare sulla superficie, è determinata esclusivamente da microrganismi presenti negli specifici ambienti di produzione e stagionatura, che causano la ricontaminazione del prodotto post-processo, possibilità legata anche al fatto che le sue caratteristiche fisico-chimiche (valore di $\left.\mathrm{pH}>6,0, \mathrm{a}_{\mathrm{w}}>0,94\right)$, non sono in grado di creare un ambiente ostile allo sviluppo dei contaminanti considerati. Cariche elevate di Escherichia coli e di Enterobacteriaceae nella ricotta assumono significato diverso rispetto alla presenza di Bacillus cereus. Il prodotto rispecchia infatti le condizioni igieniche di lavorazione e di stoccaggio e i microrganismi in esso presenti fungono pertanto da indicatori di igiene di processo. La ricerca dei tre tipi di microrganismi contaminanti aveva come obiettivo una valutazione delle caratteristiche igieniche del prodotto, con particolare riferimento a Bacillus cereus, considerato un patogeno "emergente" e che le normative comunitarie non impongono di ricercare, anche se nelle ricotte costituisce un motivo di preoccupazione per gli operatori della sanità pubblica da una parte e per i produttori dall'altra. L'indagine costituisce un primo passo verso una definita e completa valutazione di questo rischio per il consumatore.

\section{MATERIALI E METODI}

Nel periodo compreso tra Gennaio 2009 e Giugno 2012 sono stati analizzati campioni di ricotte fresche e salate, prelevati a seguito di controlli ufficiali. Il numero di campioni analizzati per anno ha seguito una distribuzione omogenea: 45 nel 2009, 41 nel 2010, 31 nel 2011 e 40 nel primo semestre del 2012, per un totale di 157 campioni, fra i quali 112 di ricotta fresca e 45 di ricotta salata. E' stata effettuata la ricerca di 3 parametri microbiologici: Bacillus cereus, Enterobacteriaceae ed Escherichia coli. La preparazione del campione prevedeva: prelievo di $10 \mathrm{~g}$ di prodotto, trasferimento in buste sterili con specifico diluente e omogenizzazione. Per ciascun parametro è stata seguita la metodica riportata nella norma ISO corrispondente: $I S O$ 21528-2 (2004) per Enterobacteriaceae; ISO 16649-2 (2001) per Escherichia coli; ISO 7932 (2005) per Bacillus cereus.

\section{RISULTATI}

I campioni analizzati sono risultati contaminati in diversa misura dai tre microrganismi considerati (tabella n. 1) La presenza di Bacillus cereus è stata riscontrata nel $53 \%$ dei campioni, con una prevalenza maggiore nelle ricotte fresche $(38 \%)$ rispetto alle salate (15\%) (grafico n.1); il $72 \%$ dei campioni contaminati presenta- va cariche $\geq 10^{3} \mathrm{UFC} / \mathrm{g}$, riscontrate soprattutto nella ricotta fresca, che in un caso ha raggiunto valori di $17 \times 10^{6} \mathrm{UFC} / \mathrm{g}$ (grafico n. 2). Le Enterobacteriaceae sono state rilevate nel $23 \%$ dei campioni analizzati, contaminando quasi nella stessa misura sia le ricotte fresche $(13 \%)$ che quelle salate $(10 \%)$. In alcuni casi, il numero di colonie ha raggiunto valori di $10^{6} \mathrm{UFC} / \mathrm{g}$ nelle ricotte fresche e di $10^{3} \mathrm{UFC} / \mathrm{g}$ nelle ricotte salate. Escherichia coli è stato riscontrato nel $12 \%$ dei campioni analizzati, con una netta prevalenza $(11 \%)$ nelle ricotte salate rispetto alle fresche $(1 \%)$.

\section{CONCLUSIONI}

Dai risultati ottenuti sembra di poter confermare che la ricotta rappresenta un prodotto particolarmente suscettibile di contaminazione, sia da parte di microrganismi indicatori di igiene di processo, come Enterobacteriaceae e Escherichia coli, sia soprattutto da microrganismi come $B a$ cillus cereus, il quale può anche produrre tossine dannose per la salute del consumatore. Secondo un parere EFSA (2005), nella maggior parte dei casi le tossinfezioni causate da Bacillus cereus sono associate a contaminazioni pari a $10^{5}-10^{8} \mathrm{UFC} / \mathrm{g}$, ma in alcuni episodi sono stati sufficienti anche quantitativi inferiori $\left(10^{3}-10^{4}\right.$ $\mathrm{UFC} / \mathrm{g}$ ). Un dato rilevante della presente ricerca è che la maggior parte dei campioni contaminati mostrava cariche superiori alla soglia di sicurezza $\left(10^{3} \mathrm{UFC} / \mathrm{g}\right)$. Occorre ricordare che la presenza di questo microrganismo nei prodotti lattiero-caseari deriva principalmente dalla contaminazione del latte nella produzione primaria ed è pertanto difficile da prevenire; il successivo trattamento termico del siero distrugge le forme vegetative di Bacillus cereus, ma non garantisce uguale risultato verso le spore, che possono resistere a temperature prossime ai $120^{\circ} \mathrm{C}$. La fase di sgrondo-raffeddamento-confezionamento, qualora avvenga troppo lentamente, rappresenta quindi il momento di maggiore rischio, in quanto il prodotto non presenta barriere efficaci verso la moltiplicazione batterica. Infatti i valori di $\mathrm{pH}$ non vanno incontro a rapido decremento, come nei formaggi, non può essere quindi ostacolata la sopravvivenza o la moltiplicazione di $B$. cereus, inoltre i valori di $\mathrm{a}_{\mathrm{w}}$ rimangono elevati e non sono previsti successivi trattamenti di risanamento. Da parte degli OSA è quindi da ritenersi necessaria una maggiore attenzione verso il processo di produzione, con l'introduzione di metodi di abbattimento rapido della temperatura per prevenire il rischio derivante da $B$. cereus e una maggiore igiene dei locali, delle attrezzature e del personale, per ridurre la ricontaminazione del prodotto ad opera di Enterobacteriaceae e Escherichia coli. 


\section{BIBLIOGRAFIA}

1. Cosentino S., Mulargia A.F., Pisano B., Tuveri P., Palmas F. 1997. Incidence and biochemical characteristics of Bacillus flora in
Sardinian dairy products. Int. J. Food Microbiol., 38, 235-238.

2. EFSA, (2005) "Bacillus cereus and other Bacillus spp in foodstuffs. EFSA Journal 175, $1-48$.

Tabella 1. Ricotte: percentuale dei risultati positivi suddivisi per anno

\begin{tabular}{|c|c|c|c|}
\hline \multicolumn{4}{|c|}{ Campioni positivi } \\
\hline & Bacillus Cereus & Enterobacteriaceae & Escherichia coli \\
\hline $\mathbf{2 0 0 9}$ & $55 \%$ & $15,5 \%$ & $22 \%$ \\
\hline $\mathbf{2 0 1 0}$ & $58 \%$ & $34 \%$ & $12 \%$ \\
\hline $\mathbf{2 0 1 1}$ & $61 \%$ & $35 \%$ & $13 \%$ \\
\hline $\mathbf{2 0 1 2}$ & $37 \%$ & $10 \%$ & $0 \%$ \\
\hline
\end{tabular}

Grafico 1. Bacillus cereus: percentuale di campioni positivi per tipologia di prodotto

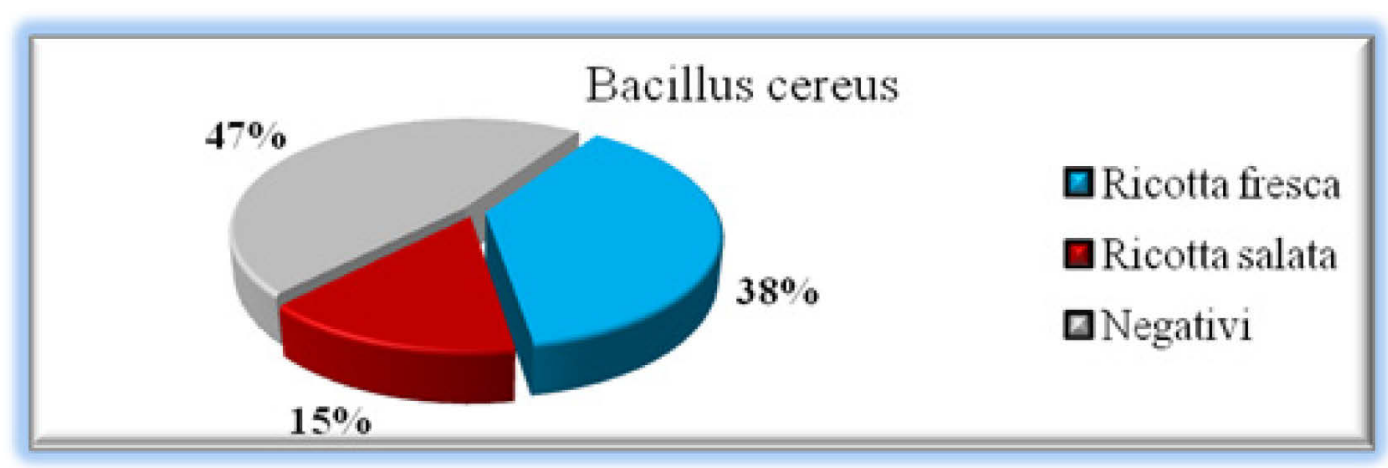

Grafico 2. B. cereus: valori percentuali dei campioni positivi suddivisi in 2 classi

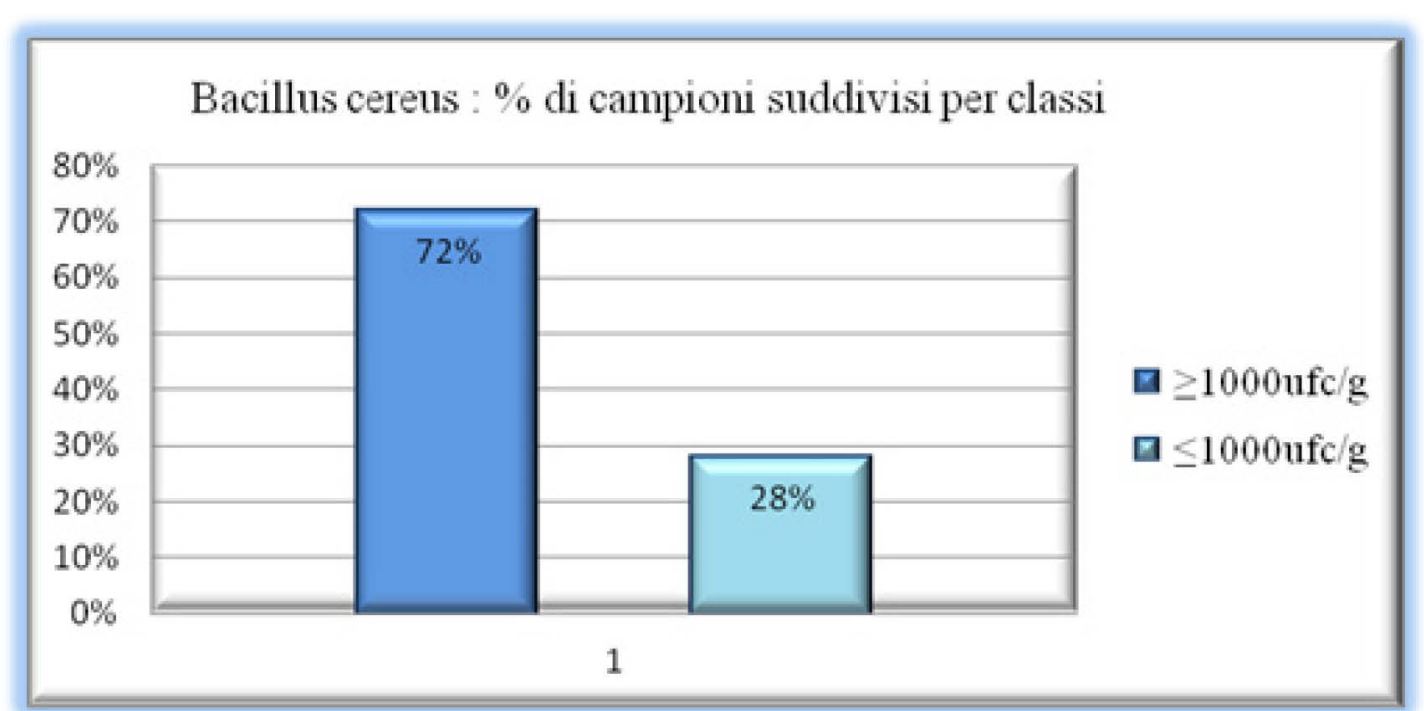

